DAMTP-R/95/29

May, 1995

\title{
INFINITE-GENUS SURFACES AND THE UNIVERSAL GRASSMANNIAN
}

\author{
SIMON DAVIS \\ Department of Applied Mathematics and Theoretical Physics \\ University of Cambridge \\ Silver Street, Cambridge CB3 9EW
}

\begin{abstract}
Correlation functions can be calculated on Riemann surfaces using the operator formalism. The state in the Hilbert space of the free field theory on the punctured disc, corresponding to the Riemann surface, is constructed at infinite genus, verifying the inclusion of these surfaces in the Grassmannian. In particular, a subset of the class of $O_{H D}$ surfaces can be identified with a subset of the Grassmannian. The concept of flux through the ideal boundary is used to study the connection between infinite-genus surfaces and the domain of string perturbation theory. The different roles of effectively closed surfaces and surfaces with Dirichlet boundaries in a more complete formulation of string theory are identified.
\end{abstract}


It is known that higher-genus correlation functions for scalar or free-fermion fields can be constructed in the operator formalism [1][2]. In this formulation, for every Riemann surface of finite genus with boundary, one may associate a state $|\phi\rangle$ in the Hilbert space based on the boundary by performing the path integral of the conformal field theory with the values of the fields specified on the boundary. The state $|\phi\rangle$ encodes information about the Riemann surfaces and one can obtain correlation functions by inserting vertex operators between $\langle 0|$, the standard vacuum in the Hilbert space, and $|\phi\rangle$. While this actually only allows the computation of the correlation functions when the positions of the vertex operators are on the disk, it is possible to analytically continue the expressions to the whole Riemann surface.

In this paper, the operator formalism is generalized for the first time to infinite-genus surfaces. These surfaces were originally discussed in the context of string theory in [3], where the total partition function is expressed as the section of a bundle on universal moduli space. While the inclusion of infinite-genus surfaces in universal moduli space suggests the presence of non-perturbative effects, it is necessary to make the formulation precise, by distinguishing between the domain of string perturbation theory and the non-perturbative contribution. This has been a central problem in string theory since the initial analyses of perturbative scattering amplitudes. It can be shown that there is a class of infinite-genus surfaces which may be included in the perturbative expansion of the vacuum amplitude known as the set of $O_{G}$ or effectively closed surfaces [4][5][6].

It therefore follows that the introduction of infinite-genus surfaces does not lead to non-perturbative effects, a result which had not yet been established at the time that the article [3] appeared. With an appropriate domain for string perturbation theory, including effectively closed infinite-genus surfaces, the perturbative expansion of the S-matrix can be defined by the vertex operator and moduli space integrals associated with these surfaces. The development of a complete formulation of string theory motivates the search for the source of non-perturbative effects. An elegant approach, recently advanced in [7][8] [9][10][11], involves the association of non-perturbative effects with the insertion of Dirichlet boundaries in the string worldsheet. 
The connection between infinite-genus surfaces and the domain of string perturbation theory is analyzed using the concept of flux through the ideal boundary and divergences in the amplitudes as vertex operators approach the boundaries. These properties can be used to distinguish between the effectively closed surfaces and the surfaces with Dirichlet boundaries. For the effectively closed surfaces, it will be demonstrated that a vanishing flux condition is satisfied, which allows for the states $|\phi\rangle$ to be solved exactly and the surfaces to be included in the universal Grassmannian in the same manner as closed finitegenus surfaces, implying that these surfaces may be used in the perturbative expansion of the S-matrix. Support for the association of non-perturbative effects with surfaces having Dirichlet boundaries shall come from the behaviour of the correlation function as the vertex operators approach the boundaries. The different roles of the effectively closed surfaces and the surfaces with Dirichlet boundaries, therefore, can be established in a more complete formulation of string theory.

In [1], it was shown that for the free scalar field theory on a Riemann surface,

$$
S=\int_{\Sigma-D} X \partial \bar{\partial} X
$$

there are an infinite number of symmetries

$$
X \rightarrow X+\epsilon_{n} X_{n}
$$

where $X_{n}$ represents a perturbation which corresponds to a function with a pole of order n at $t=0$ that is harmonic in $\Sigma-D$ (Fig. 1). 


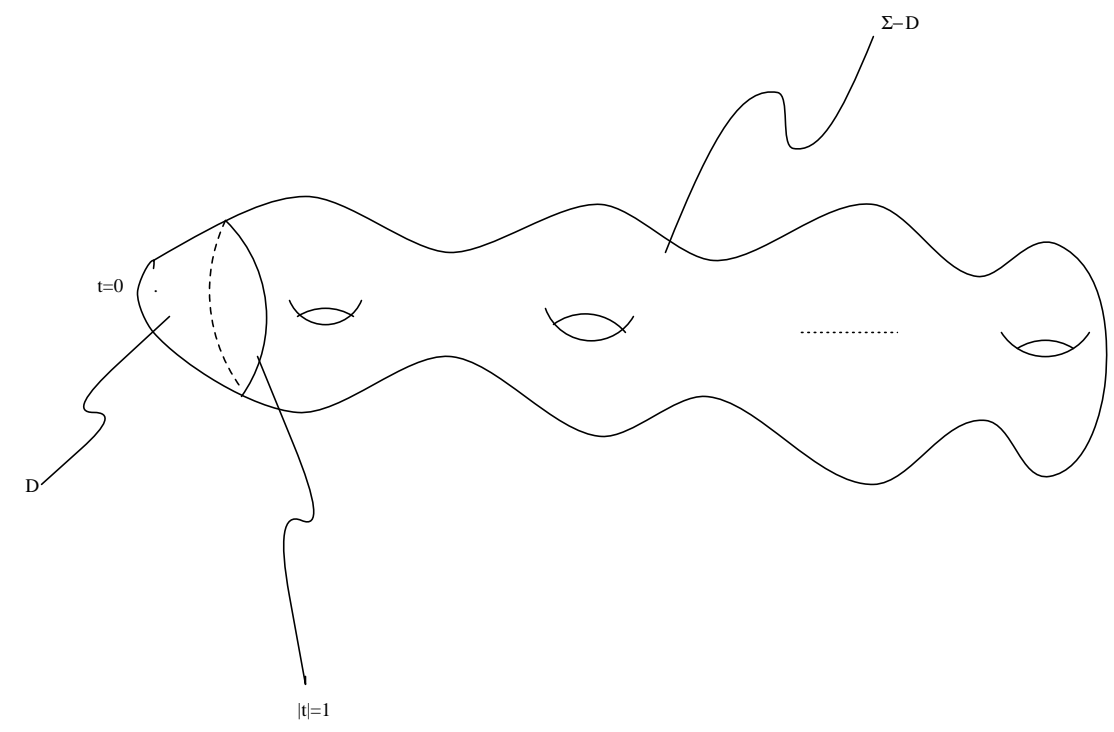

Fig. 1. Splitting of a Riemann surface into a disc D and the genus-g complement $\Sigma-D$.

The action becomes

$$
\int_{\Sigma-D} X \partial \bar{\partial} X+\epsilon_{n} \int_{\Sigma-D} \partial\left(X_{n}^{a} \bar{\partial} X\right)+\epsilon_{n} \int_{\Sigma-D} \bar{\partial}\left(X_{n}^{h} \partial X\right)=\int_{\Sigma-D} X \partial \bar{\partial} X+\epsilon_{n} Q_{n}
$$

where

$$
Q_{n}=\int_{S}\left(X_{n}^{a} \bar{\partial} X+X_{n}^{h} \partial X\right)
$$

with $X_{n}^{h}$ and $X_{n}^{a}$ being the holomorphic and anti-holomorphic parts of $X_{n}$ respectively. It is only when the scalar field $\mathrm{X}$ satisfies the equations of motion and is therefore harmonic that the decomposition $X=X^{h}(z)+X^{a}(\bar{z})$ is valid, simplifying the formula for the charge $Q_{n}$. The path integral does not change if

$$
Q_{n}|\phi\rangle=0
$$

This leads to an infinite number of conditions which are integrable because the charges $Q_{n}$ commute. The state $|\phi\rangle$ can be obtained within a constant factor.

This argument made use of Stokes' theorem. Now consider the planar covering of a finite-genus surface with boundary S (Fig. 2) 


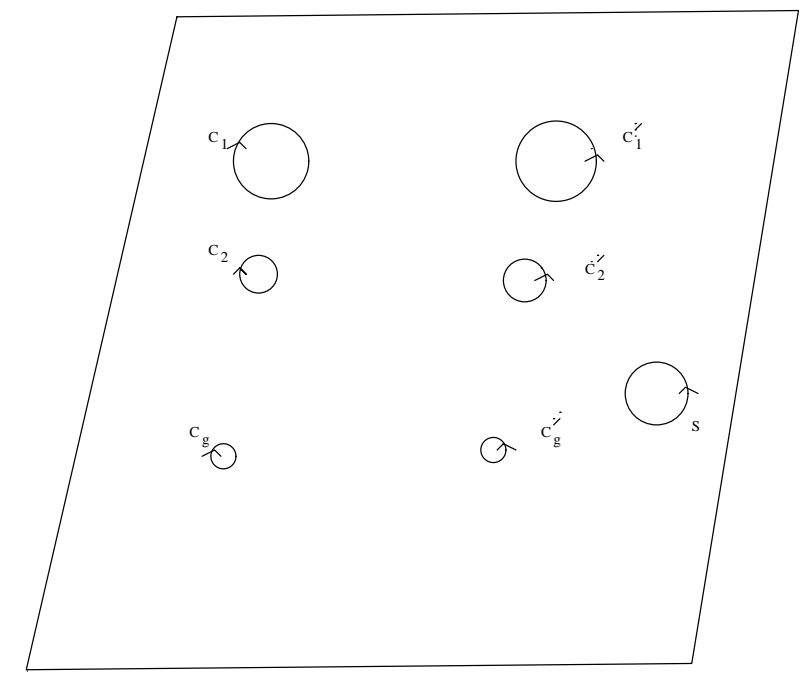

\section{Fig. 2 Cancellation of contour integration on the Schottky covering of a Rie- mann surface.}

One sees that the boundary of the fundamental region is $S \cup \cup_{n=1}^{g}\left(C_{n} \cup C_{n}^{\prime}\right)$, the union of two distinct sets, $\mathrm{S}$ and $\cup_{n=1}^{g}\left(C_{n} \cup C_{n}^{\prime}\right)$, where the latter set represents the $2 \mathrm{~g}$ isometric circles of the Schottky group uniformizing the surface. The differential on the Riemann surface is now replaced by a differential on the plane which is automorphic under the action of the Schottky group, $\omega(\Gamma z)=\omega(z)$. However, the contours $C_{n}$ and $C_{n}^{\prime}$ are oriented in opposite directions. Consequently, the line integrals over them vanish and one is left with a line integral over S.

There also exist points in the universal Grassmannian, defined to be the set of rays in the Hilbert space of states for the free field theory on the punctured disc, corresponding to infinite-genus surfaces, and one may wish to solve for the state $|\phi\rangle$ using the method applied to finite-genus surfaces. The use of Stokes' theorem implies that one would have to consider the line integral on $\mathrm{S}$ and the integral on the ideal boundary (Fig. 3). 


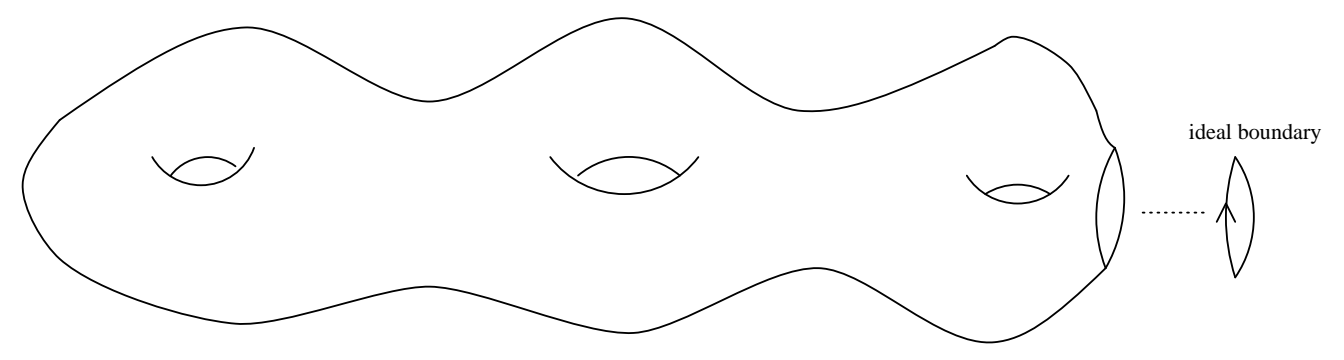

Fig. 3 Contour integration over the ideal boundary of a Riemann surface.

For integrability, it is necessary to impose the condition

$$
\int_{\substack{\text { ideal } \\ b d y}} j_{n}=0
$$

where $j_{n}=X_{n}^{a} \bar{\partial} X+X_{n}^{h} \partial X$ is the current. The vanishing of the integral at the ideal boundary is needed to satisfy the operator condition $Q_{n}|\phi\rangle=0(5)$ as $Q_{n}=\int j_{n}$. The simplest way to achieve this result follows by requiring that $X_{n}$ vanishes at the boundary for each $n$.

Recalling that for compact surfaces, by the Weierstrass gap theorem, there are g values of $\mathrm{n}$ between 1 and $2 \mathrm{~g}$ for which there does not exist a meromorphic function with a pole of order $\mathrm{n}$ at some point. This problem is circumvented by allowing $X_{n}$ to have holomorphic and anti-holomorphic parts [1].

The Weierstrass gap problem does not occur for infinite-genus surfaces. Every divisor on a non-compact surface is the divisor of a meromorphic function. In fact, given an arbitrary discrete set of points on the surface, one can find a meromorphic function which has poles of any order that is selected for these points. This follows from the BehnkeStein theorem [12] which shows that there exists an everywhere regular analytic function with any discrete set of zeros. The order of these zeros can be chosen arbitrarily. If $f$ is such an analytic function, then $\frac{1}{f}$ is a meromorphic function with the required properties. Moreover, it is possible that $f$ is unbounded in the approach to the ideal boundary. Then $\frac{1}{f}$ would tend to zero. So, it may be possible to find meromorphic functions $X_{n}$, with poles of order $\mathrm{n}$, tending to zero at the ideal boundary, for each $\mathrm{n}$. This would imply that the 
state $|\phi\rangle$ would be a simple tensor product of a state with holomorphic degrees of freedom and a state with anti-holomorphic degrees of freedom.

Thus, defining the universal Grassmannian to be the space of such vacuum states, up to a multiplicative factor, every compact finite-genus surface and certain infinite-genus surfaces will correspond to points in the Grassmannian. Moreover, the one-to-one correspondence between the standard Fock vacuum and the vacuum state for the Riemann surface could be regarded as being equivalent to the formulation of the axiom of asymptotic completeness in Euclidean quantum gravity, which is required for the factorization of the $\$$ matrix [13]. This one-to-one correspondence between the vacua implies that unitarity of the free fermion theory is maintained in the infinite-genus limit. The extension to string theory might be established by consideration of the full string action.

An example of an infinite-genus surface which could be included in the universal Grassmannian would be the sphere with an infinite number of handles studied in [5]. One may recall that the correlation function can be calculated explicitly using the method of images.

$$
\begin{aligned}
G\left(z_{P} ; z_{R}, z_{S}\right)= & \sum_{\alpha} \ln \left|\frac{z_{P}-V_{\alpha} z_{R}}{z_{P}-V_{\alpha} z_{S}}\right| \\
& \quad-\frac{1}{2 \pi} \sum_{m, n=1}^{\infty} \operatorname{Re}\left(v_{n}\left(z_{P}\right)\right)(\operatorname{Im} \tau)_{m n}^{-1} \operatorname{Re}\left(v_{n}\left(z_{R}\right)-v_{n}\left(z_{S}\right)\right)
\end{aligned}
$$

where

$$
v_{n}(z)=\sum_{\alpha}{ }^{(n)} \ln \left(\frac{z-V_{\alpha} \xi_{1 n}}{z-V_{\alpha} \xi_{2 n}}\right)
$$

is holomorphic on the the fundamental domain since $\xi_{1 n}, \xi_{2 n}$ are fixed points of $T_{n}$ and $\sum_{\alpha}(n)$ includes only those $V_{\alpha}$ for which $T_{n}, T_{n}^{-1}$ is not the right-most member of the element $V_{\alpha}$. It may be noted that in the second term, the definition of $v_{n}(z)$ requires convergence of the Poincare series [5]. While the period matrix is infinite-dimensional, it still has eigenvalues

$$
(\operatorname{Im} \tau) \tilde{e}_{n}=\lambda_{n} \tilde{e}_{n}
$$

where $\tilde{e}_{n}$ is the nth eigenvector, which can also be written as $\tilde{e}_{n}=U e_{n}$ with $e_{n}$ being the nth unit vector in $\mathbb{R}^{\infty}$. As $U^{-1}(\operatorname{Im} \tau) U=\operatorname{diag}\left(\lambda_{1}, \ldots, \lambda_{n}, \ldots\right)$, 
$(\operatorname{Im} \tau)^{-1}=U \operatorname{diag}\left(\lambda_{1}^{-1}, \ldots, \lambda_{n}^{-1}, \ldots\right) U^{-1}$ if the eigenvalues are non-zero. The existence of $U^{-1}$ requires that the column vectors of $\mathrm{U}$, eigenvectors of $(\operatorname{Im} \tau)$, be linearly independent. This follows since the complement to the space spanned by the eigenvectors is the kernel, which will have non-zero dimension if the eigenvectors are linearly dependent. However, if $\operatorname{dim} \operatorname{ker}(\operatorname{Im} \tau) \neq 0$, the determinant of $\operatorname{Im} \tau$ vanishes, contrary to the assumption that the eigenvalues are non-zero.

Positivity of the eigenvalues of $\operatorname{Im} \tau$ generally follows from the bilinear relation

$$
(\omega, \sigma)=\sum_{n=1}^{g}\left[\int_{A_{n}} \omega \int_{B_{n}} \bar{\sigma}-\int_{A_{n}} \sigma \int_{B_{n}} \bar{\omega}\right]
$$

A generalized bilinear relation holds on infinite-genus surfaces in the class $O_{H D}$ [14], which, by definition, admit no non-constant harmonic functions with finite Dirichlet norm. To verify this property for open surfaces can be quite involved, and it is easier to use the following result [15]:

Theorem. Let $p_{0}, p_{1}$ be principal functions for any pairs of logarithmic singularities $\log \left|z-z_{R}\right|$ and $-\log \left|z-z_{S}\right|$. If $p_{0}-p_{1}$ is constant, then the surface is in $O_{H D}$.

The principal functions are harmonic everywhere except for the singularities, with $p_{0}$ having zero normal derivative and $p_{1}$ having vanishing flux on the ideal boundary. This theorem will now be used for surfaces which can be uniformized by groups of Schottky type.

To the Green function (7), one may add a harmonic function of the form $\sum_{n} a_{n} \sum_{\alpha} \operatorname{Re} v_{n}\left(V_{\alpha} z\right)+C$. The normal derivative at a point on a circle of radius $\mathrm{r}$ is

$$
\begin{aligned}
\frac{1}{2} \sum_{n} a_{n} & \sum_{\alpha}\left(\left.e^{i \theta} \frac{d v_{n}(u)}{d u}\right|_{u=V_{\alpha} z}+\text { c.c. }\right) \\
& =\frac{1}{2} \sum_{n} a_{n} \sum_{\alpha}\left(e^{i \theta} \sum_{\beta}{ }^{(n)} \frac{V_{\beta} \xi_{1 n}-V_{\beta} \xi_{2 n}}{\left(V_{\alpha} z-V_{\beta} \xi_{1 n}\right)\left(V_{\alpha} z-V_{\alpha} \xi_{2 n}\right)}+\text { c.c. }\right)
\end{aligned}
$$

If $\infty$ is a limit point of the uniformizing Schottky group $\Gamma$, invariance of the limit point set $\Gamma$ implies that there exist elements $V_{\alpha}$ such that $V_{\alpha} z=V_{\beta} \xi_{1 n}$ or $V_{\alpha} z=V_{\beta} \xi_{2 n}$. These 
two terms are infinite but they cancel. Thus, the terms giving the dominant contribution are those for which $V_{\alpha} z \in I_{V_{\beta}^{-1}}$, requiring $V_{\alpha}=V_{\beta} V_{\gamma}^{\prime}$ where the left-most members of $V_{\gamma}^{\prime}$ do not form the element $V_{\beta}^{-1}$.

$$
\begin{aligned}
\sum_{\gamma}{ }^{(\beta)} \sum_{\beta}{ }^{(n)} \frac{V_{\beta} \xi_{1 n}-V_{\beta} \xi_{2 n}}{\left(V_{\beta} V_{\gamma}^{\prime} z-V_{\beta} \xi_{1 n}\right)\left(V_{\beta} V_{\gamma}^{\prime} z-V_{\beta} \xi_{2 n}\right)}= \\
\sum_{\gamma}{ }^{(\beta)} \sum_{\beta}{ }^{(n)} \frac{\left(\xi_{1 n}-\xi_{2 n}\right) \gamma_{\beta}^{2}}{\frac{\left(V_{\gamma}^{\prime} z-\xi_{1 n}\right)}{\left(V_{\gamma}^{\prime} z+\frac{\delta_{\beta}}{\gamma_{\beta}}\right)} \frac{\left(V_{\gamma}^{\prime} z-\xi_{2 n}\right)}{\left(V_{\gamma}^{\prime} z+\frac{\delta_{\beta}}{\gamma_{\beta}}\right)}}
\end{aligned}
$$

As z tends to $\infty, V_{\gamma}^{\prime} z \equiv \frac{\alpha_{\gamma} z+\beta_{\gamma}}{\gamma_{\gamma} z+\delta_{\gamma}}$ tends to $\frac{\alpha_{\gamma}}{\gamma_{\gamma}}$ so that the terms in (11) become constant. Since the circle at infinity represents the ideal boundary, defined to be the limit set of $\Gamma$ factored by $\Gamma$, the normal derivative vanishes at the boundary only if each $a_{n}$ is zero. The flux is formally obtained by integrating the normal derivative around the circle at infinity, which could be viewed as potentially representing the ideal boundary of a non-compact surface. Expanding the sum in (12) in powers of $\frac{1}{z}, c_{0 n}+\frac{c_{1 n}}{z}+\frac{c_{2 n}}{z^{2}}+\ldots$ and using $\int_{0}^{2 \pi} r e^{i \theta} c_{0} d \theta=0, \lim _{r \rightarrow \infty} \int_{0}^{2 \pi} r e^{i \theta} \frac{c_{m n}}{z^{m}} d \theta=0$ for $m \geq 2$, one sees that the contribution of $(12)$ to the flux is $2 \pi c_{1 n}$ where

$$
\begin{aligned}
c_{1 n}=\sum_{\gamma}{ }^{(\beta)} \sum_{\beta}{ }^{(n)}\left(\xi_{1 n}-\xi_{2 n}\right) \gamma_{\beta}^{2}\left(\frac{\alpha_{\gamma}}{\gamma_{\gamma}}+\frac{\delta_{\beta}}{\gamma_{\beta}}\right) \frac{\beta_{\gamma}-\delta_{\gamma}}{\gamma_{\gamma}} \frac{1}{\left(\frac{\alpha_{\gamma}}{\gamma_{\gamma}}-\xi_{1 n}\right)} \\
\frac{1}{\left(\frac{\alpha_{\gamma}}{\gamma_{\gamma}}-\xi_{2 n}\right)}\left[2-\left(\frac{\alpha_{\gamma}}{\gamma_{\gamma}}+\frac{\delta_{\beta}}{\gamma_{\beta}}\right)\left[\frac{1}{\left(\frac{\alpha_{\gamma}}{\gamma_{\gamma}}-\xi_{1 n}\right)}+\frac{1}{\left(\frac{\alpha_{\gamma}}{\gamma_{\gamma}}-\xi_{2 n}\right)}\right]\right]
\end{aligned}
$$

Thus, again, to obtain vanishing flux, one requires that all $a_{n}$ vanish. It follows that the difference between the principal functions $p_{0}-p_{1}$ is a constant and the surface is in the class $O_{H D}$.

Since $v_{n}(z)$ is an automorphic function on the complex plane, $\sum_{\alpha} \operatorname{Re} v_{n}\left(V_{\alpha} z\right)$ diverges and should only be regarded as a formal expression. Alternatively, one may consider the functions

$$
h_{n}(z, \bar{z})=\sum_{\alpha} \sum_{p} \operatorname{Re}\left[v_{n}\left(V_{\alpha} z\right)-v_{n}\left(V_{\alpha} z_{0}\right)\right] b_{p}\left(V_{\alpha} z-V_{\alpha} z_{0}\right)^{p}
$$


where each term is finite for $p \geq 1$ because of the convergence of the Poincare series $\sum_{\alpha \neq I}\left|\gamma_{\alpha}\right|^{-2}<\infty$. Thus

$$
\begin{aligned}
h_{n}^{\prime}(z, \bar{z}) & =\left.\sum_{\alpha} \sum_{p} \frac{d}{d u}\left[\left[\operatorname{Re}\left(v_{n}(u)\right)-\operatorname{Re}\left(v_{n}\left(V_{\alpha}\left(z_{0}\right)\right)\right)\right] b_{p}\left(u-V_{\alpha} z_{0}\right)^{p}\right]\right|_{u=V_{\alpha} z} \\
& =\sum_{\alpha} \sum_{p} \sum_{\beta}{ }^{(n)} \operatorname{Re}\left[\frac{V_{\beta} \xi_{1 n}-V_{\beta} \xi_{2 n}}{\left(V_{\alpha} z-V_{\alpha} \xi_{1 n}\right)\left(V_{\alpha} z-V_{\alpha} \xi_{2 n}\right)}\right] b_{p}\left(V_{\alpha} z-V_{\alpha} z_{0}\right)^{p} \\
& +\sum_{\alpha} \sum_{p}\left[\operatorname{Re}\left(v_{n}\left(V_{\alpha} z\right)-v_{n}\left(V_{\alpha} z_{0}\right)\right)\right] p b_{p}\left(V_{\alpha} z-V_{\alpha} z_{0}\right)^{p-1}
\end{aligned}
$$

and

$$
\begin{aligned}
h_{n}^{\prime}(z, \bar{z}) & \rightarrow \sum_{z \rightarrow \infty} \sum_{p} b_{p}\left(z-z_{0}\right)^{p} \sum_{\beta}{ }^{(n)} \operatorname{Re}\left[\frac{V_{\beta} \xi_{1 n}-V_{\beta} \xi_{2 n}}{\left(z-V_{\beta} \xi_{1 n}\right)\left(z-V_{\beta} \xi_{2 n}\right)}\right] \\
& +\sum_{p} p b_{p}\left(z-z_{0}\right)^{p-1}\left[\operatorname{Re}\left(v_{n}(z)-v_{n}\left(z_{0}\right)\right)\right]
\end{aligned}
$$

implying divergence of the integral

$\iint_{F . D .}\left|h_{n}^{\prime}(z, \bar{z})\right|^{2} d z \wedge d \bar{z} \rightarrow \sum_{r=0}^{\infty} \tilde{b}_{r}\left(\operatorname{Re}\left(v_{n}\left(z_{0}\right)\right)^{2} \iint_{F . D .-\Delta}\left|z-z_{0}\right|^{r-2} d z \wedge d \bar{z}+\right.$ finite

with F.D. being the fundamental domain of the uniformizing group, $\Delta$ being a region of finite size and $\tilde{b}_{r}=\sum_{p+q=r} p q b_{p} b_{q}$. If this property is valid for all non-constant harmonic functions on the Riemann surface, then it belongs to the class $O_{H D}$. The category of Riemann surface can also be determined by the normal derivative and the flux for the harmonic functions $h_{n}(z, \bar{z})$ of equation (14). By analogy with equation (12), the normal 
derivative in the limit $z \rightarrow \infty$ is

$$
\begin{aligned}
& \frac{1}{2} \sum_{n} a_{n} e^{i \theta} h_{n}^{\prime}(z)+c . c . \\
& =\frac{1}{2} \sum_{n} a_{n} e^{i \theta} \sum_{p} \sum_{\gamma}(\beta) \sum_{\beta}{ }^{(n)} \frac{\left(\xi_{1 n}-\xi_{2 n}\right) \gamma_{\beta}^{2-2 p} b_{p}\left(\frac{\alpha_{\gamma}}{\gamma_{\gamma}}-V_{\gamma}^{\prime} z_{0}\right)^{p}}{\left(\frac{\alpha_{\gamma}}{\gamma_{\gamma}}-\xi_{1 n}\right)\left(\frac{\alpha_{\gamma}}{\gamma_{\gamma}}-\xi_{2 n}\right)\left(\frac{\alpha_{\gamma}}{\gamma_{\gamma}}+\frac{\delta_{\beta}}{\gamma_{\beta}}\right)^{p-2}\left(V_{\gamma}^{\prime} z_{0}+\frac{\delta_{\beta}}{\gamma_{\beta}}\right)^{p}} \\
& +\frac{1}{2} \sum_{n} a_{n} e^{i \theta} \sum_{p} \sum_{\gamma}{ }^{(\beta)} \sum_{\beta}{ }^{(n)} \sum_{\delta}(n) \ln \left|\frac{\frac{\alpha_{\gamma}}{\gamma_{\gamma}}-V_{\delta} \xi_{1 n}}{\frac{\alpha_{\gamma}}{\gamma_{\gamma}}-V_{\delta} \xi_{2 n}} \frac{V_{\gamma}^{\prime} z_{0}-V_{\delta} \xi_{2 n}}{V_{\gamma}^{\prime}-V_{\delta} \xi_{1 n}}\right| \\
& \cdot p b_{p}\left[\frac{\left(\frac{\alpha_{\gamma}}{\gamma_{\gamma}}-V_{\gamma}^{\prime} z_{0}\right)}{\left(\frac{\alpha_{\gamma}}{\gamma_{\gamma}}+\frac{\delta_{\beta}}{\gamma_{\beta}}\right)\left(V_{\gamma}^{\prime} z_{0}+\frac{\delta_{\beta}}{\gamma_{\beta}}\right)}\right]^{p-1} \\
& \gamma_{\beta}^{2-2 p}+c . c .
\end{aligned}
$$

As each of the terms multiplying the coefficients $a_{n}$ are non-vanishing constants, the normal derivative is zero only if each of the $a_{n}$ is zero. Note that the sums multiplying $a_{n}$ will only be finite when $b_{0}=b_{1}=0$ and $\sum_{\beta}{ }^{(n)} \gamma_{\beta}^{2-2 p}<\infty$ only when $p \geq 2$.

The flux is $2 \pi \sum_{n} a_{n} c_{1 n}$ where, by analogy with equation (13),

$$
\begin{aligned}
c_{1 n}=\sum_{\gamma}{ }^{(\beta)} \sum_{\beta}{ }^{(n)} & \frac{\gamma_{\beta}^{-2 p}}{\left(z_{0}+\frac{\delta_{\beta}}{\gamma_{\beta}}\right)^{p}}\left(\frac{\alpha_{\gamma}}{\gamma_{\gamma}}+\frac{\delta_{\beta}}{\gamma_{\beta}}\right)^{-(p-1)}\left(V_{\gamma}^{\prime} z_{0}+\frac{\delta_{\beta}}{\gamma_{\beta}}\right)^{-p} \\
& \cdot \frac{1}{\left(\frac{\alpha_{\gamma}}{\gamma_{\gamma}}-\xi_{1 n}\right)} \frac{1}{\left(\frac{\alpha_{\gamma}}{\gamma_{\gamma}}-\xi_{2 n}\right)} \\
& {\left[\frac{\beta_{\gamma}-\delta_{\gamma}}{\gamma_{\gamma}}\left[2-\left(\frac{\alpha_{\gamma}}{\gamma_{\gamma}}+\frac{\delta_{\beta}}{\gamma_{\beta}}\right)\left[\frac{1}{\left(\frac{\alpha_{\gamma}}{\gamma_{\gamma}}-\xi_{1 n}\right)}+\frac{1}{\left(\frac{\alpha_{\gamma}}{\gamma_{\gamma}}-\xi_{2 n}\right)}\right]\right]\right.} \\
& \left.-p\left[\left(z_{0}+\frac{\delta_{\beta}}{\gamma_{\beta}}\right)\left(\frac{\alpha_{\gamma}}{\gamma_{\gamma}}+\frac{\delta_{\beta}}{\gamma_{\beta}}\right)+\frac{\beta_{\gamma}-\gamma_{\gamma}}{\delta_{\gamma}}\right]\right]
\end{aligned}
$$

Actually, by Green's theorem, the flux for a harmonic function equals the Dirichlet norm, and therefore, the sums in (19) should diverge. The flux vanishes only when $a_{n}=0$ for all $\mathrm{n}$, and consequently the difference between the principal functions $p_{0}$ and $p_{1}$ must be a constant, implying that the surface is in $O_{H D}$.

Indeed, it has been proven that surfaces uniformized by a group of Schottky type are in the class $O_{G}[4]$, characterized by the property that the ideal boundary has zero linear 
measure. The model considered here is useful for consideration of both $O_{G}$ surfaces and the surfaces with ideal boundary at infinity, as the isometric circles are clearly tending towards a point $\infty$ in the extended complex plane, but this may also be regarded as the infinite radius limit of a sequence of circles, with each circle representing a closed curve on the Riemann surface. The analysis above, derived for surfaces uniformized by groups of Schottky type, and therefore applicable to $O_{G}$ surfaces, provides a useful indication of the calculation of the flux for the more general $O_{H D}$ surfaces. The restriction to the $O_{G}$ surfaces can be implemented by taking the radius of the limit circle to be zero, and one may notice that this factor is cancelled in the calculation of the flux so that its magnitude does not affect the results mentioned above.

The same method can be applied to the Green function (7) for $O_{G}$ surfaces uniformized by Schottky groups. One may also recall that the existence of a Green function with two sources at $z_{R}$ and $z_{S}$, with behaviour $\ln \left|z_{P}-z_{R}\right|$ and $-\ln \left|z_{P}-z_{S}\right|$ can be demonstrated for more general Riemann surfaces [16]. In the limit $z_{P} \rightarrow \infty, G\left(z_{P} ; z_{R}, z_{S}\right)$ and $\frac{\partial G}{\partial z_{P}}$ tend to zero. Similarly, one may wish to calculate the flux at the accumulation point of the isometric circles from this Green function. The study of the principal functions shows that this quantity also vanishes. It is now possible to make an identification of a subset of $O_{H D}$ with a subset of the Grassmannian. A necessary condition for the solvability of equation (5) on open surfaces with ideal boundary is the vanishing of the flux (6). Those surfaces in $O_{H D}$ for which this condition is sufficient will belong to the Grassmannian because an exact solution for $|\phi\rangle$ has been obtained. Conversely, those surfaces corresponding to points in the Grassmannian satisfy the vanishing flux condition and therefore possess Green functions with two sources with vanishing normal derivative at the ideal boundary if they also lie in $O_{H D}$.

One may note a similarity with the analysis of Green and Polchinski [9][10][17] regarding the insertion of boundaries in string worldsheets. It is of interest to note that a divergence in the correlation function arises, for the surfaces that they study, as the locations of the vertex operators near the boundary [8]. This divergence confirms the interpretation of the boundary states as point-particle states [7][8]. The absence of similar divergences 
in the computation of the four-point function [5] on a sphere with an infinite number of handles is consistent with the absence of flux through the accumulation point and the assumption that there are no additional sources which may be associated with boundary states. Consequently, the effectively closed surfaces naturally may be included in the perturbative expansion of the S-matrix, whereas the surfaces with Dirichlet boundaries inserted contribute to non-perturbative effects in string theory.

\section{Acknowledgements}

The vanishing flux condition at the ideal boundary, required for the application of the operator formalism to infinite-genus surfaces, was initially formulated at the Lyman Laboratory in 1988. I would like to thank Prof. S. W. Hawking and Dr. G. W. Gibbons for their support while this paper has been completed. 


\section{REFERENCES}

[1] C. Vafa, Phys. Lett. $\underline{190 B}$ (1987) $47-54$

[2] L. Alvarez-Gaume, C. Gomez and C. Reina, Phys. Lett. 190B (1987) 55 - 62

L. Alvarez-Gaume, C. Gomez, G. Moore and C. Vafa, Nucl. Phys. B303 (1988) 455 521

[3] D. Friedan and S. Shenker, Phys. Lett. $\underline{B 175}$ (1986) 287 - 296

D. Friedan, Physics and Mathematics of Strings :

Memorial Volume for Vadim Knizhnik, ed. by L. Brink, D. Friedan and

A. M. Polyakov (Singapore: World Scientific, 1990)

[4] S. Davis, Class. Quantum Grav. $\underline{6}$ (1989) 1791 - 1803

[5] S. Davis, Mod. Phys. Lett. A $\underline{9}(14)$ (1994) 1299 - 1307

[6] S. Davis, J. Math. Phys. $\underline{36}(2)$ (1995) 648 - 663

[7] M. B. Green, Phys. Lett. $\underline{B 266}$ (1991) 325 - 336

[8] M. B. Green, Phys. Lett. $\underline{B 329}$ (1994) 435 - 443

[9] M. B. Green and J. Polchinski, Phys. Lett. $\underline{B 335}$ (1994) 377 - 382

[10] J. Polchinski, Phys. Rev. D50 (1994) 6041 - 6045

[11] M. B. Green, 'A Gas of D-Instantons', hep-th/9504108

[12] O. Forster, Lectures on Riemann Surfaces (New York: Springer-Verlag, 1981)

[13] S. W. Hawking, Commun. Math. Phys. $\underline{87}$ (1982) 395 - 415

[14] R. D. M. Accola, Trans. Amer. Math. Soc., $\underline{96}$ (1960) 143

Y. Kusonoki, Mem. Coll. Sci. Univ. Kyoto, Series A Math. 30(1) 1 
[15] L. Ahlfors and L. Sario, Riemann Surfaces (Princeton: Princeton University Press, 1960)

[16] H. Farkas and I. Kra, Riemann Surfaces (New York: Springer-Verlag, 1980)

[17] M. B. Green and P. Wai, Nucl. Phys. $\underline{B 431}$ (1994) 131 - 172 